\title{
Elevated TAK1 augments tumor growth and metastatic capacities of ovarian cancer cells through activation of NF-KB signaling
}

\author{
Patty C.H. Cai ${ }^{1}$, Lei Shi ${ }^{2}$, Vincent W.S. Liu ${ }^{1}$, Hermit W.M. Tang ${ }^{1}$, Iris J. Liu ${ }^{1}$, Thomas \\ H.Y. Leung ${ }^{1}$, Karen K.L. Chan ${ }^{1}$, Judy W.P. Yam ${ }^{3}$, Kwok-Ming Yao ${ }^{2}$, Hextan Y.S. \\ Ngan ${ }^{1}$ and David W. Chan ${ }^{1}$ \\ ${ }^{1}$ Department of Obstetrics and Gynaecology, LKS Faculty of Medicine, The University of Hong Kong, Hong Kong SAR, \\ P.R.China \\ 2 Department of Biochemistry, LKS Faculty of Medicine, The University of Hong Kong, Hong Kong SAR, P.R.China \\ ${ }^{3}$ Department of Pathology, LKS Faculty of Medicine, The University of Hong Kong, Hong Kong SAR, P.R.China \\ Correspondence to: Hextan YS Ngan, email: hysngan@hku.hk \\ David W Chan, email: dwchan@hku.hk
}

Keywords: TAK1, NF-KB signaling, ovarian cancer, high-grade tumor

Received: May 07, $2014 \quad$ Accepted: July 26, $2014 \quad$ Published: July 27, 2014

This is an open-access article distributed under the terms of the Creative Commons Attribution License, which permits unrestricted use, distribution, and reproduction in any medium, provided the original author and source are credited.

\section{ABSTRACT}

Transforming growth factor (TGF)- $\beta$-activating kinase 1 (TAK1) is a serine/ threonine kinase which is frequently associated with human cancer progression. However, its functional role in tumorigenesis is still controversial. Here, we report that TAK1 enhances the oncogenic capacity of ovarian cancer cells through the activation of NF-KB signaling. We found that TAK1 is frequently upregulated and significantly associated with high-grade and metastatic ovarian cancers. Mechanistic studies showed that Ser412 phosphorylation is required for TAK1 in activating NF-KB signaling and promotes aggressiveness of ovarian cancer cells. Conversely, suppression of TAK1 activity by point mutation at Ser412, RNAi mediated gene knockdown or TAK1 specific inhibitor ((5Z) -7-Oxozeaenol) remarkably impairs tumor growth and metastasis in ovarian cancer in vitro and in vivo. Our study underscores the importance of targeting TAK1 as a promising therapeutic approach to counteract the ovarian cancer progression.

\section{INTRODUCTION}

Ovarian cancer is one of the leading causes of cancer death in females worldwide [1]. The high mortality rate of this disease is due to its late diagnosis and therefore poor prognosis as most cases present with aggressive ovarian cancer [2]. Despite advances in cancer treatment in the past decade, the cure rate of this disease remains modest [3]. Recent studies have revealed that personalized gene-targeted cancer therapy shows promising results in improving the survival and quality of life of patients suffering from aggressive tumors [4-7]. Therefore, a better understanding of the molecular mechanisms underlying ovarian cancer oncogenesis is urgently needed for guiding this therapeutic approach.

It is well-known that nuclear factor-kappa B
(NF- $\mathrm{KB}$ ) signaling pathway has multiple roles in cancer progression such as anti-apoptosis, cell cycle, angiogenesis and metastasis [8]. However, its mechanistic roles in human cancers are varied because it regulates the expression of over 400 genes that are simultaneously stimulated by multiple upstream regulators [9]. Degradation of IкBs through the proteasomal pathway after phosphorylation by IKKs activates NF- $\mathrm{\kappa B}$ signaling activity. Thus, $\mathrm{p}$-IKK and $\mathrm{p}$-I $\mathrm{KB}$ can be monitored to reflect NF- $\kappa B$ signaling activity [10]. Previous studies have stated that blockage of NF- $\mathrm{KB}$ activity increases the efficiency of chemotherapy by cisplatin in ovarian cancer models [11] and suppresses ovarian cancer cell metastasis [12]. Hence, the NF- $\mathrm{kB}$ signaling pathway is a promising target in cancer therapy.

Transforming growth factor (TGF)- $\beta$-activating 
kinase 1 (TAK1) is a serine/threonine kinase and a mitogen-activated protein kinase kinase kinase that can be activated by many upstream cytokines, such as TGFbeta, IL-1beta, TNF-alpha, and toll-like receptor ligands [13]. Previous reports have shown that TAK1 may play diverse roles in various cancers by acting as tumor suppressor or oncoprotein. For instance, in liver cancer, ablation of TAK 1 can cause hepatic injury, inflammation, fibrosis and carcinogenesis, which identifies TAK1 as a tumor suppressor [14]. However, in skin tumors, pancreatic cancers and colon cancers, inhibition of TAK1 up-regulates ROS, sensitizes cells to chemoresistance, and promotes apoptosis, respectively [15-17]. Indeed, the functional roles and molecular mechanisms of TAK1 in ovarian cancers remain obscure. Effects of TAK1 are mediated via phosphorylation of multiple residues in its activation loop. Many phosphorylation sites had been previously identified, such as Ser-192, Thr178, Thr184/187 and Ser412 [18-21] but only Thr184/Thr187 has been well studied in promonocytic leukemic and prostate cancer cells $[22,23]$. However, which phosphorylated site required for TAK1 mediated $\mathrm{NF}-\kappa \mathrm{B}$ signaling in ovarian oncogenesis is still unclear.
In this study, we provide compelling evidence showing TAK1 is frequently overexpressed in aggressive and high-grade ovarian cancer. Importantly, TAK1 activates NF- $\kappa$ B signaling activity through the increased phosphorylation at Ser412, and such activated TAK1/ $\mathrm{NF}-\kappa \mathrm{B}$ signaling cascade is indispensable in promoting ovarian cancer cell growth, anchorage independent growth ability, chemoresistance, as well as in vitro and in vivo metastasis in ovarian cancer. These results reveal mechanistic insights into the functional role of TAK 1 in $\mathrm{NF}-\kappa \mathrm{B}$ mediated ovarian cancer aggressiveness, suggesting TAK1 is a therapeutic target for this disease.

\section{RESULTS}

\section{TAK1 is frequently upregulated in ovarian cancer}

To understand the functional role and expression status of TAK1 in ovarian cancer, qPCR analysis was performed to evaluate the expression level of TAK1 mRNA in ovarian cancer samples $(n=88)$, normal ovaries
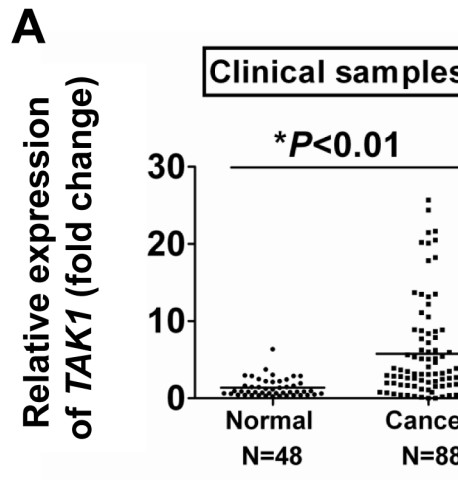

C

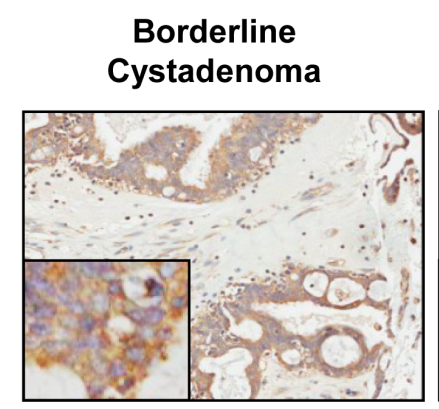

B

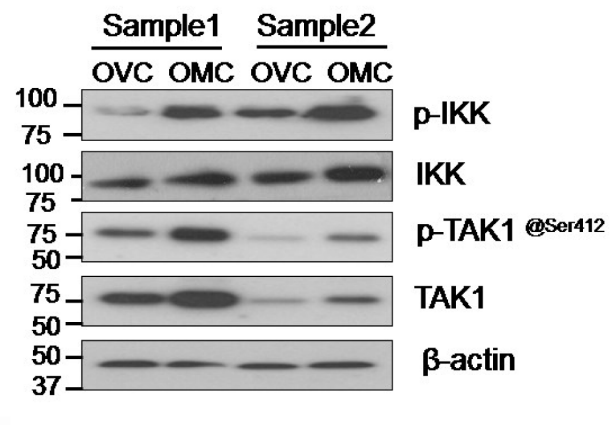

IHC: anti-TAK1

Figure 1: TAK1 is upregulated in high-grade and metastatic ovarian cancer. (A) Q -PCR using $M A P 3 K$ probe was performed for three times independently in normal cancer samples $(n=48)$, ovarian cancer samples $(n=88)$, HOSE cell lines $(n=2)$ and ovarian cancer cell lines $(\mathrm{n}=6)$. The expression of TAK1 mRNA was normalized by internal control $18 S$ gene. $* P<0.01$. (B) Representative IHC showed the staining intensity of TAK1 in borderline cystadenoma, serous low-grade and high-grade on an ovarian cancer tissue array (OVC1021) (x20). Increased immune-positive staining of TAK1 was observed along low- to high-grade ovarian cancers. (C) Western blot analysis using anti-TAK1, p-TAK1 at Ser412, IKK and p-IKK (Ser180/181) of 2 pairs of primary cultured cells from omentum (OMC) and ovary (OVC). The protein amount of loading was normalized by $\beta$-actin. 
$(n=48)$, normal ovarian HOSE cell lines $(n=2)$ and ovarian cancer cell lines $(n=6)$. The results showed that TAK1 was significantly upregulated in ovarian cancer samples by 8 -fold and ovarian cancer cell lines by 18 -fold as compared with normal ovaries and ovarian HOSE cell lines, respectively $(* P<0.01)$ (Figure $1 \mathrm{~A})$. Clinicopathological correlation indicated that overexpresson of TAK1 was remarkably associated with high-grade tumor formation ( ${ }^{*} P=0.033$ ) (Supplementary Table 1$)$. However, there was no significant association between TAK1 overexpression and other clinical parameters. Furthermore, Western blot and immunohistochemical analysis were conducted to evaluate the protein expression level of TAK1 in ovarian cancer cell lines and a commercial tissue array (OV1021, Pantomics) respectively. Results showed that the expression of TAK1 was obviously upregulated in ovarian cancer cell lines as compared to the HOSEs (Supplementary Figure S1). In addition, there is a progressive increase in TAK1 expression from lowgrade to high-grade serous ovarian cancers (Figure 1B). By clinicopathological correlation analysis, high TAK1 expression was significantly correlated with high-grade tumor again $(P=0.001)$, in which $47.9 \%$ of high-grade tumor cases exhibited more than 6-fold overexpression of TAK 1 whereas $85.4 \%$ of low-grade tumor cases showed lower expression of TAK1 (Supplementary Table 2). In addition, expression of TAK1 was highly correlated with cancer cell metastasis $(P=0.025)$, in which $54 \%$ of cases demonstrated more than 6-fold overexpression of TAK1 (Supplementary Table 2). Most of serous ovarian cancers are found to have omental metastasis [24]. This implies that the cancer cells from the omentum are considered more aggressive as compared from the cancer cells still contained within the ovary. Thus, we cultured the primary ovarian cancer cells from both omentum and ovary of the same ovarian cancer patient $(n=2)$. Western blot analysis showed that there was a remarkable upregulation of TAK1, p-TAK1 at Ser412 and p-IKK (Ser180/181) in primary ovarian cancer cells from omentum (OMC) as compared with primary ovarian cancer cells from the ovaries (OVC) (Figure 1C). The p-TAK1 (Ser412) and p-IKK (Ser180/181) represent the activities of TAK1 and $\mathrm{NF}-\kappa \mathrm{B}$ respectively. Therefore, this result supports the findings from clinicopathological analysis, suggesting that increased TAK1 and NF- $\mathrm{KB}$ signaling activities are involved in aggressive ovarian cancer cells.

\section{TAK1 promotes ovarian cancer cell growth and anchorage independent growth ability}

Given that TAK1 was frequently overexpressed in ovarian cancer, in particular high-grade tumor, study of the functional role of TAK1 in ovarian cancer cells is very worthwhile. Stable TAK1-overexpressing clones were generated by transfection of TAK1 plasmids into ovarian cancer cell lines; OVCA429 (429-C12 and 429C13) and A2780cp (Acp-T2 and Acp-T3), while stable knockdown of endogenous TAK1 was achieved in SKOV3 (SK-sh1-KD3 and SK-sh1-KD6) and A2780cp (Acp-sh1KD1 and Acp-sh2-KD10) cells using vector-based RNAi constructs (Figure 2A-B). By XTT cell proliferation assay, enforced expression of TAK1 in OVCA429 and A2780cp showed an average 5-fold and 2-fold, respectively, higher proliferation rate than their vector controls $(* P<0.01)$ (Figure 2A). In contrast, depletion of TAK1 in SKOV3 and A2780cp decreased the proliferation rate by $\sim 30 \%$ and $\sim 50 \%$, respectively, when compared to their vector controls $(* P<0.01)$ (Figure $2 \mathrm{~B})$. To further prove the importance of TAK1 in ovarian cancer cell growth, the specific TAK1 inhibitor, (5Z) -7-Oxozeaenol, was used to treat A2780cp and SKOV3 and the TAK1-overexpressing OVCA429 clones 429-C12 and 429-C13. XTT cell proliferation assay showed that inhibitor treatment suppressed the proliferation of A2780cp, SKOV3, and both $429-\mathrm{C} 12$ and $429-\mathrm{C} 13$, by $\sim 66 \%, \sim 75 \%$, and $\sim 80 \%$, respectively, when compared with the untreated controls $(* P<0.01)$ (Figure 2C). In addition, soft agar assay showed that enforced expression of TAK 1 increased not only the size but also the number of colonies in Acp-T2 and Acp-T3 by 1.5 -fold and 1.2 -fold, respectively $(* P<0.01)$. On the other hand, depletion of TAK 1 reduced both the size and number of colonies in SK-sh1-KD3 and SK-sh1-KD6 by $\sim 40 \%$ and $\sim 70 \%$, respectively, $(* P<0.01)$ as compared with their vector controls (Figure 2D). Furthermore, focus formation assay demonstrated that the TAK1-overexpressing clones in OVCA429 (429-C12 and 429-C13) and A2780cp (Acp-T2 and Acp-T3) exhibited more and larger colonies by $\sim 1.5$-fold and $\sim 2$-fold, respectively, when compared with their vector controls $(* P<0.05)$ (Figure 2E). In contrast, the stable TAK1 knockdown clones in SKOV3 (SK-sh1-KD3 and SK-sh1-KD6) and A2780cp (Acp-sh1-KD1 and Acp-sh2-KD10) showed less and smaller colonies by $\sim 50 \%$ and $\sim 66 \%$, respectively, as compared with their vector controls $(* P<0.05)$ (Figure $2 \mathrm{~F})$. Taken together, these results indicate that TAK1 is capable of promoting ovarian cancer cell growth and anchorage independence.

\section{TAK1 increases tumor growth in vivo in ovarian cancer cells}

In order to investigate the tumor forming capacity of TAK1 in ovarian cancer cells, the stable TAK1 knockdown clone (Acp-sh1-KD1) and vector control of A2780cp were selected for subcutaneous injection into nude mice at each side of flank. Acp-sh1-KD1 exhibited reduced tumor growth rate by $60 \%$ when compared to the vector control ( $* P=0$. 022) (Figure $2 \mathrm{G}$ ). Western blot analysis confirmed that the tumor tissues from Acp-KD1treated mice expressed lower levels of TAK1, p-IKK and 
A
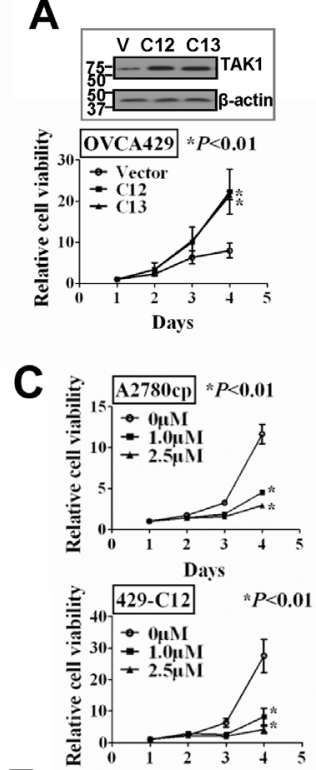

E
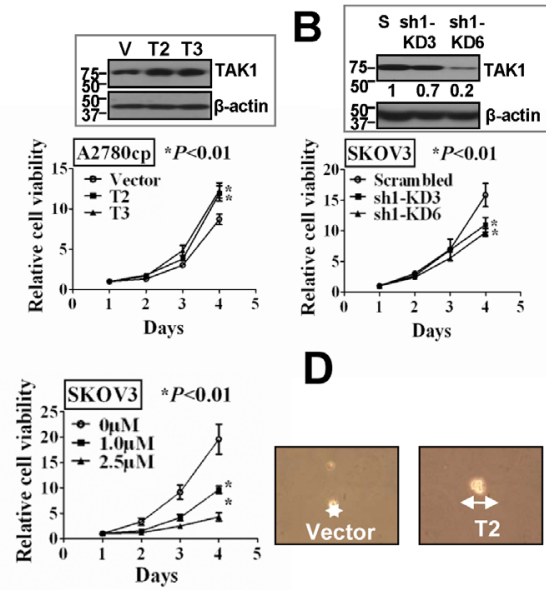

D
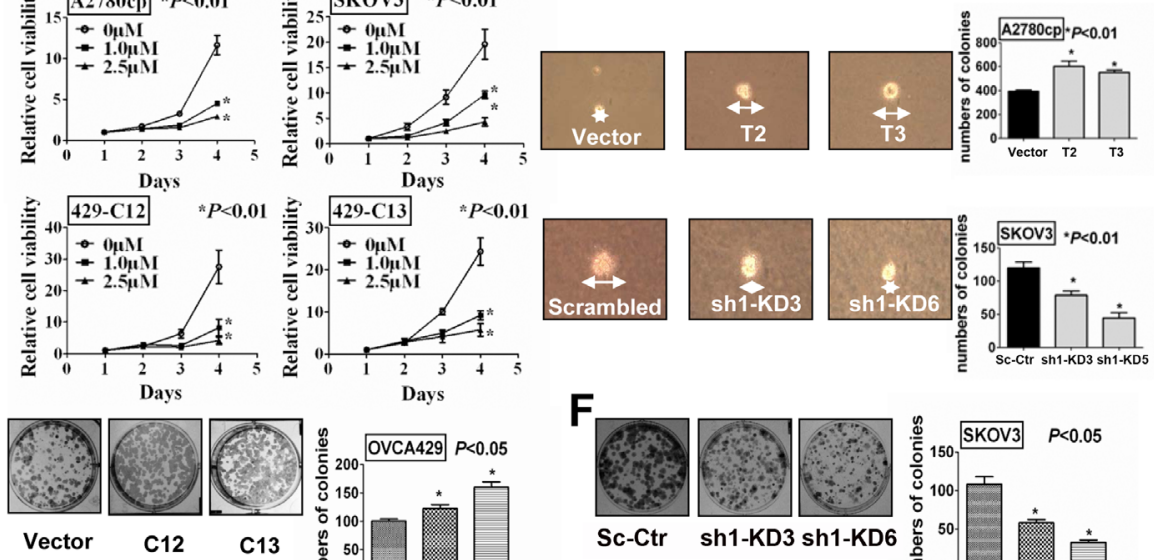

$\mathbf{F}$
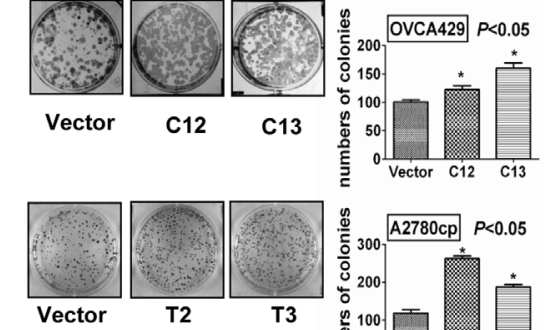

$\mathbf{G}$
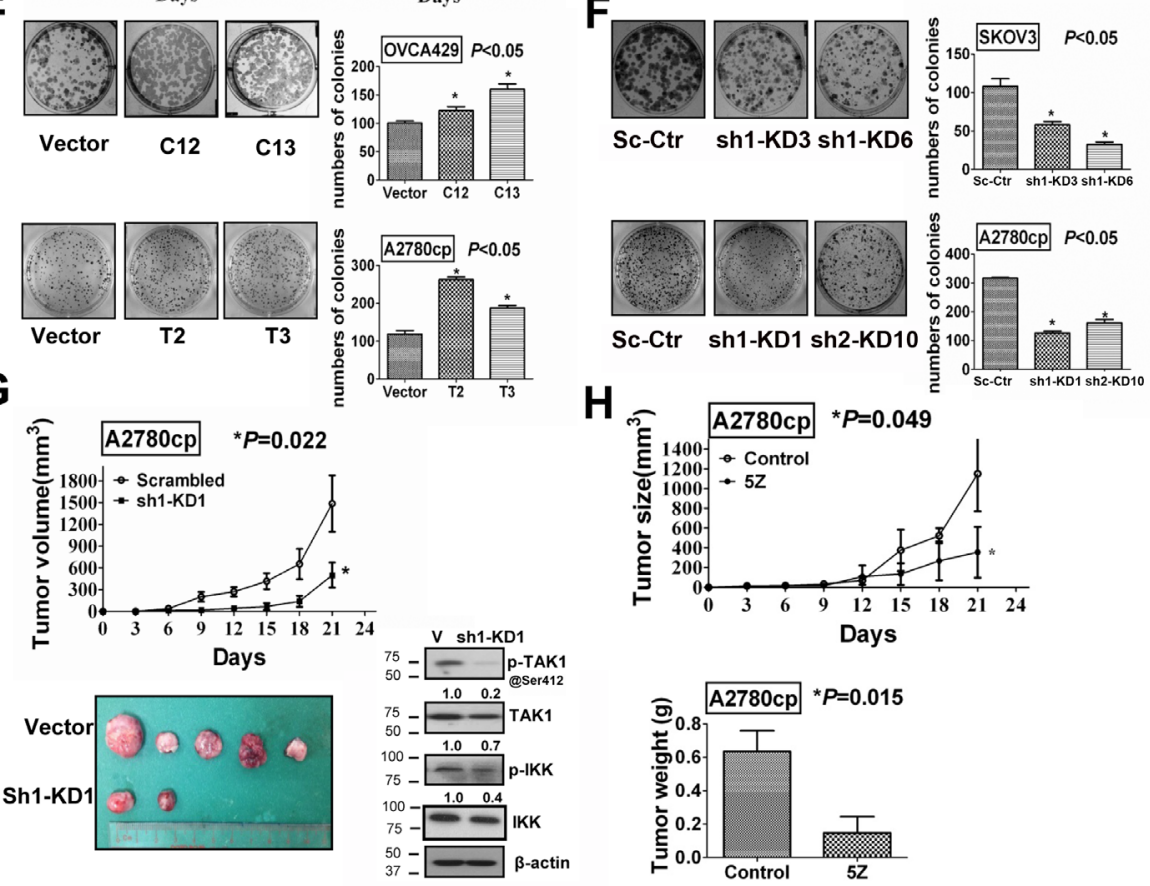

$\mathbf{H}$
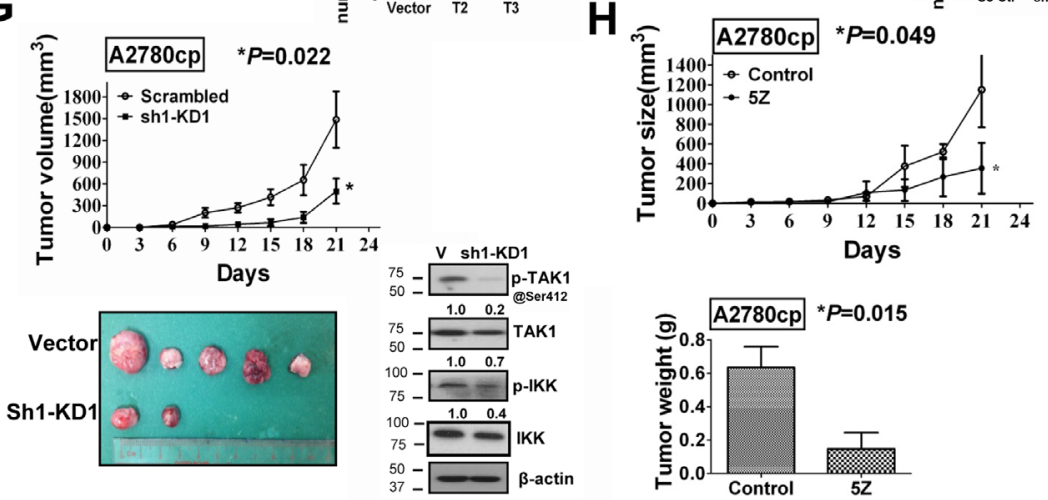

Figure 2: TAK1 promotes ovarian cancer cell growth in vitro and in vivo. (A) Stable TAK1 over-expressing clones were established in OVCA429 and A2780cp cells. XTT cell proliferation assay demonstrated that TAK1 could remarkably increase cell proliferation in OVCA429 and A2790cp stable clones $\left({ }^{*} P<0.01\right)$. (B) TAK1 knockdown stable clones were established by shRNA in SKOV3 and A2780cp cells. Depletion of endogenous TAK1 reduced cell proliferation in SKOV3 and A2790cp stable clones $(* P<0.01)$. (C) Treatment of TAK1 inhibitor, (5Z) -7-Oxozeaenol, (1.0 $\mu \mathrm{M}$ and $2.5 \mu \mathrm{M}$ for 24 hours) significantly reduced cell proliferation in both ovarian cancer parental cell lines (A2780cp and SKOV3) $\left({ }^{*} P<0.01\right)$ and TAK1 stably over-expressing clones $(429-\mathrm{C} 12$ and $429-\mathrm{C} 13)$ $(* P<0.01)$ dose dependently. (D) Soft agar assay showed that TAK1-expressing clones exhibited an increased number of colonies compared with the vector control $(* P<0.01)$, whereas clones with depletion of TAK1 had less number of colonies as compared with the vector control $\left({ }^{*} P<0.01\right)$. Representative photos show the size of colonies under microscopy. (E) Enforced expression of TAK1 in OVCA429 and A2780cp significantly increased the number of cell colonies, $(* P<0.05)$. (F) Depletion of TAK1 by shRNA knockdown in SKOV3 and A2780cp decreased the number of cell colonies $(* P<0.05)$. (G) Stable TAK1 knockdown clone (Acp-sh1-KD1) showed a slower growth rate as compared with the vector control in nude mouse tumor xenograft model $(* P=0.022)$. Representative tumor picture was taken after sacrificed the nude mice on Day 21. Western blot analysis confirmed the expressions of TAK1, p-TAK1 at Ser412, p-IKK and IKK from the tumor tissues. (H) TAK1 inhibitor, $(5 \mathrm{Z})-7-$ Oxozeaenol $(16 \mathrm{mg} / \mathrm{kg})$, significantly inhibited the tumor formation rate in nude mice as compared with the parental A2780cp cell control $(* P=0.049)$. The weight of the tumors treated with TAK 1 inhibitor was approximately 3 -folds lower as compared with control group $(* P=0$. 015). 
p-TAK1 at Ser412 (Figure 2G). To further confirm TAK1 in tumor growth of ovarian cancer cells, A2780cp cells expressing high levels of TAK1 were injected into nude mice subcutaneously in the presence and absence of (5Z) -7-Oxozeaenol treatment. Starting on day $3,16 \mathrm{mg} / \mathrm{kg}(5 \mathrm{Z})$ -7-Oxozeaenol or PBS (carrier solution) control, was intraperitoneally injected (i.p.) for every three days in each group of tumor bearing nude mice $(n=5)$. After 7 times of injection, we found that there was $\sim 70 \%$ reduction in tumor volume with (5Z) -7-Oxozeaenol treatment when compared with the PBS control on Day21 ( $P=0$. 049) (Figure 2H). Besides, the average tumor weight on Day21 with (5Z) -7-Oxozeaenol injection was $\sim 75 \%$ lower as compared with the PBS control group $(* P=0.015)$ (Figure $2 \mathrm{H})$. Altogether, these data further support the notion that overexpression of TAK 1 contributes to ovarian cancer cell growth in vivo.

A
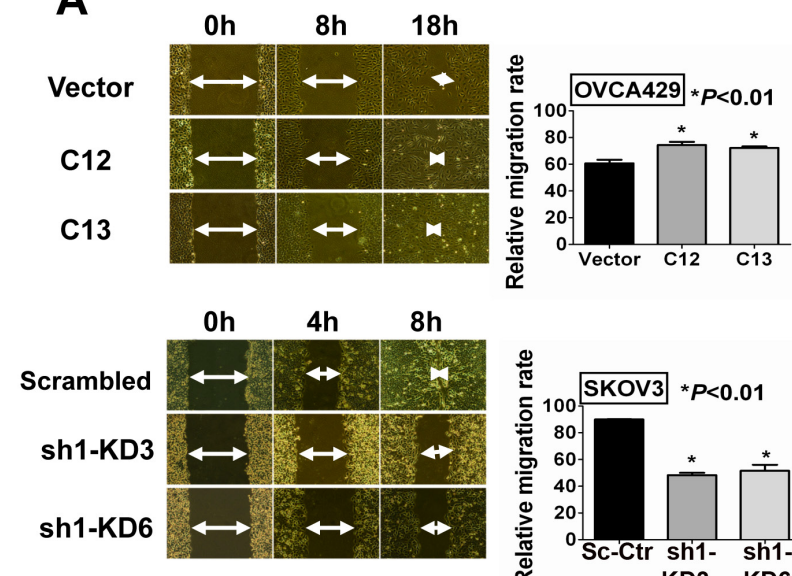

C

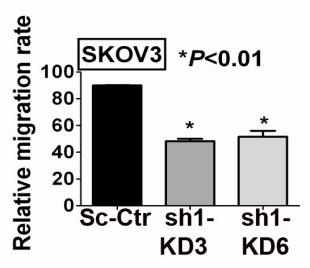

SKOV3
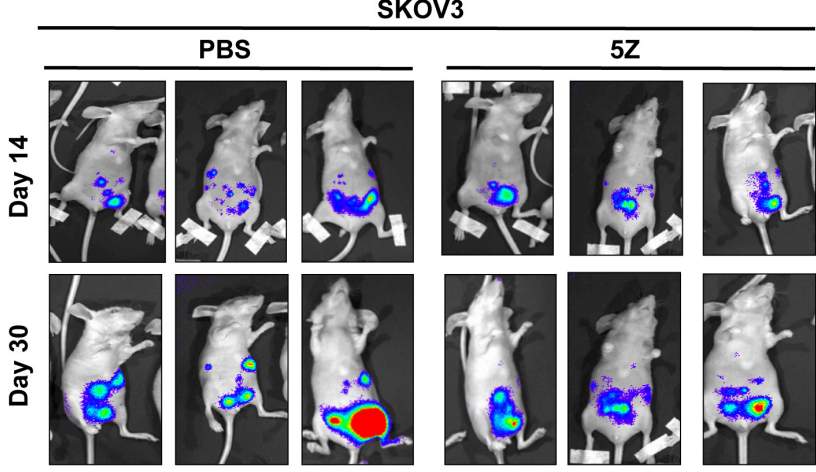

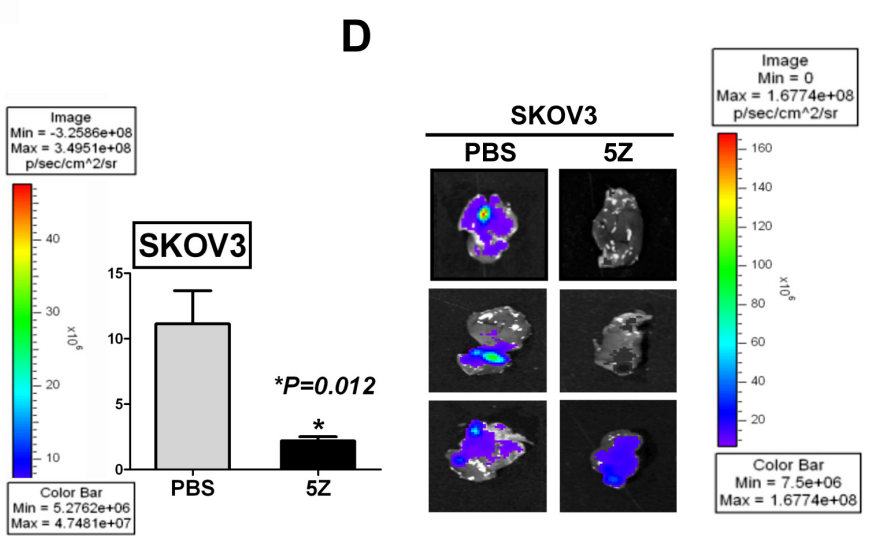

$24 h$

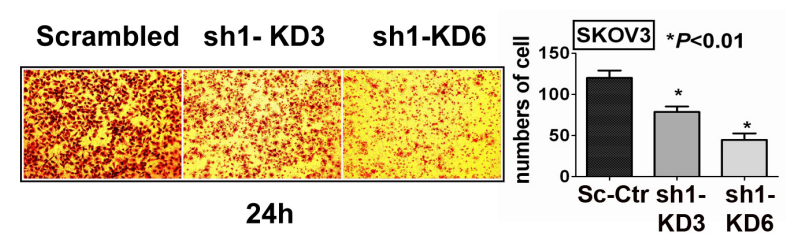

\section{TAK1 promotes cell migration/invasion in vitro} and in vivo of ovarian cancer cells

High cell proliferation, migration and invasion are salient features of aggressive high-grade ovarian tumors[25]. On the other hand, previous studies have shown that TAK1 is required for bone metastasis [26] and inhibition of TAK1 blocks cancer cell invasion and metastasis in breast cancer[27]. Hence, we postulated that TAK 1 overexpression is able to promoting cell migration and invasion of ovarian cancer cells. Wound healing assay was firstly performed to examine the function of TAK1 in the cell migration capacity of ovarian cancer cells. Upon treatment of Mitomycin $\mathrm{C}$ to exclude the factor of increased cell growth, we observed a faster wound closure rate in $429-\mathrm{C} 12$ and $429-\mathrm{C} 13$ by 1.4 -fold and 1.3 fold, respectively, when compared to their vector controls $(* P<0.01)$. Conversely, knockdown of endogenous TAK1

B

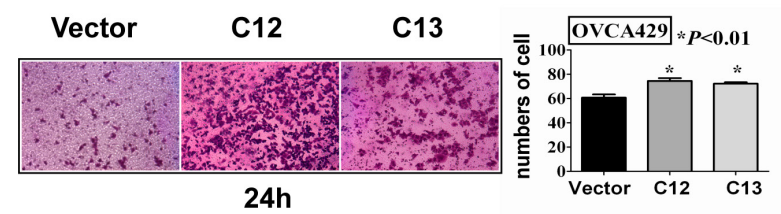

Figure 3: TAK1 enhances ovarian cancer cell migration/invasion in vitro and in vivo. (A) Wound healing assay showed the over-expressed TAK1 exhibited a faster wound closure rate in 429-C12 and 429-C13 $\left({ }^{*} P<0.01\right)$, while knockdown of TAK1 exhibited a lower wound closure rate in SK-KD3 and SK-KD6 $\left({ }^{*} P<0.01\right)$ as compared with their vector controls. The arrows indicate the width of the wound and the relative cell migration rate is expressed as relative width of the wounds/time by bar charts. (B) Transwell cell invasion assay and the bar chart showed a higher invasive rate through Matrigel-coated membrane in TAK1 stably over-expressing clones (Acp-T2 and Acp-T3) $\left({ }^{*} P<0.01\right)$, while a lower invasive rate was observed in TAK1 knockdown clones SK-KD3 and SK-KD6 $\left({ }^{*} P<0.01\right)$ when compared to their vector controls. (C) Representative bioluminescence images on Day14 and Day30 of nude mice in PBS (carrier solution) group and TAK1 inhibitor ((5Z) -7-Oxozeaenol, $16 \mathrm{mg} / \mathrm{kg}, 5$ injections) group; PBS (carrier solution) group ( $\mathrm{n}=3)$ presented significant higher relative bioluminescence index on Day30 as compared to the TAK1 inhibitor group ( $\mathrm{n}=3$ ), $P=0.012$; (D) Representative biofluorosence imaging of the livers. On Day30 for SKOV3 control group with PBS (carrier solution) and TAK1 inhibitor treatment group. 
in SK-sh1-KD3 and SK-sh1-KD6 significantly reduced the cell migration rate by $50 \%$ and $40 \%$, respectively, as compared with their vector controls $(* P<0.01)$ (Figure 3A). Furthermore, using Transwell invasion assays, we demonstrated that there was a remarkable increase by 1.5 -fold and 1.3-fold in cell invasion rate in Acp-T2 and Acp-T3, respectively, as compared with vector controls $(* P<0.01)$. In contrast, the numbers of cells invading through matrigel in Transwell invasion assays were significantly reduced in SK-sh1-KD3 and SK-sh1KD6 by $30 \%$ and $60 \%$, respectively, as compared with vector controls $(* P<0.01)$ (Figure $3 \mathrm{~B})$. To further confirm these functional roles of TAK1, a mouse model for in vivo study of ovarian cancer metastasis was conducted. The GFP-luminescence labelled SKOV3 cells (CMVGFP-T2A-Luciferase) were injected (intraperitoneally) i.p. into 6 nude mice. After 14 days, bioluminescence images were taken to record the start point (Figure 3C). Then the mice were separated into two groups; one group received intraperitoneal injections of TAK1 inhibitor, (5Z) -7-Oxozeaenol $(16 \mathrm{mg} / \mathrm{kg})$, while the control group was injected with PBS only. After 5 injections, the bioluminescence imaging of the PBS group displayed prominent tumor size growth with an average 10-fold increase, whereas only 3.2-fold increase could be observed in the TAK 1 inhibitor treated group on day $30(* P=0.012)$ (Figure $3 \mathrm{C}$ ). In addition, the livers of the mice were collected at the end of the experiment and examined with biofluorosence. Results showed that the livers in PBS group demonstrated stronger intensity of GFP signal as compared to the TAK1 inhibitor group, indicating that the TAK1 inhibitor could inhibit SKOV3 cells metastasized to livers of mice (Figure 3D). Taken together, these data suggest that inhibition of TAK1 activity is able to impair
A

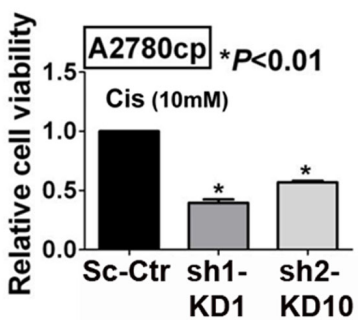

C

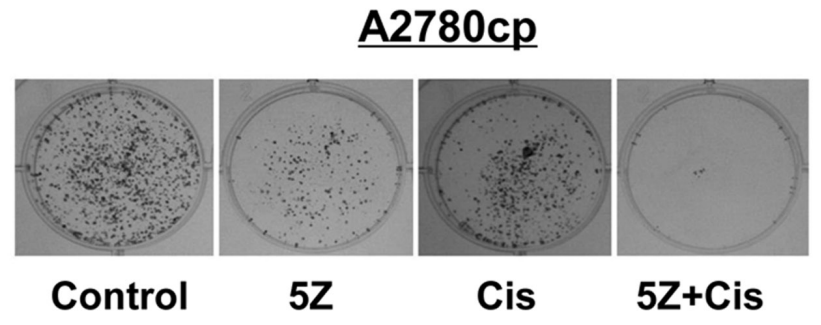

ES-2

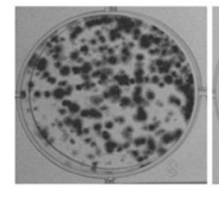

Control

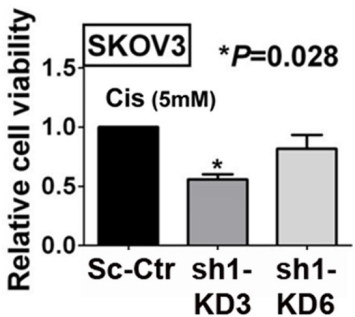

KD3 KD6
Cis

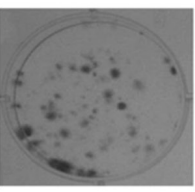

$5 Z$

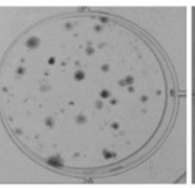

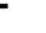

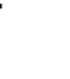

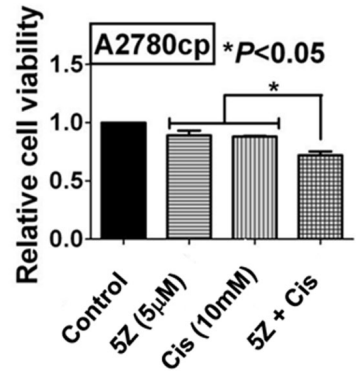
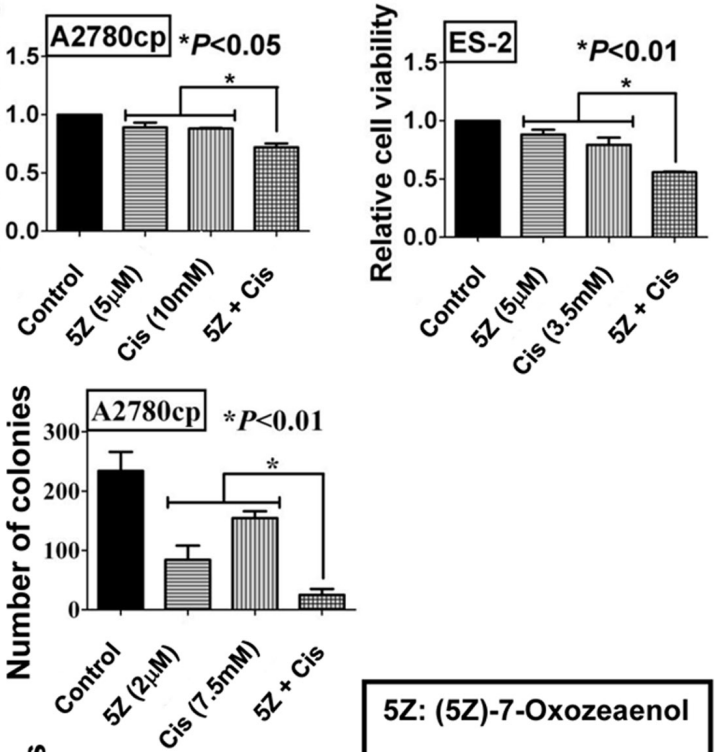

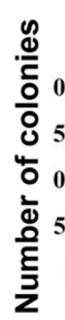

$5 Z+C$ is

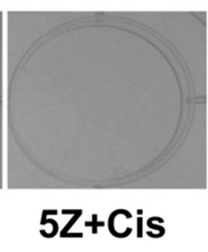

Figure 4: Inhibition of TAK1 sensitizes ovarian cancer cells to cisplatin-induced cell apoptosis. (A) XTT proliferation assay was performed to detect the effect of cisplatin on cell proliferation with ovarian cancer cells. The bar chart shows that TAK1 knockdown stable clones were more sensitive to cisplatin treatment as compared to vector control. Stable clones Acp-KD1 and Acp-KD10 were treated with $10 \mathrm{mM}$ cisplatin for $48 \mathrm{~h}(* P<0.01)$, and SK-KD3 and SK-KD6 were treated with $5 \mathrm{mM}$ cisplatin for $48 \mathrm{~h}\left(\mathrm{C} 3,{ }^{*} P=0.028\right)$. (B) The bar chart showed that TAK1 inhibitor, $(5 \mathrm{Z})-7-O x o z e a e n o l(5 \mu \mathrm{M})$, could sensitize ovarian cancer A2780cp $(* P<0.05)(\mathrm{cisplatin}: 10 \mathrm{mM})$ and ES-2 $(* P<0.01)$ (cisplatin: $3.5 \mathrm{mM})$ to cisplatin-induced cell apoptosis. The results were obtained from XTT cell proliferation assay in a 48-hour time course. (C) Focus formation assay was performed and showed that TAK1 inhibitor, (5Z) -7-Oxozeaenol (2 $\mu \mathrm{M})$, could sensitize ovarian cancer A2780cp $(* P<0.01)$ (cisplatin: $7.5 \mathrm{mM})$ and ES-2 $(* P<0.01)$ (cisplatin: $2 \mathrm{mM})$ to cisplatin-induced cell apoptosis as compared with their controls or each single treatment. Representative photos were taken to show the number and size of the colonies. 
in vitro and in vivo ovarian cancer cell motility and metastasis.

\section{TAK1 sensitizes ovarian cancer cells to cisplatin- induced cytotoxicity}

Previous studies have reported that TAK1 increases chemoresistance via the NF- $\mathrm{KB}$ pathway and targeting TAK1 has been shown to be a potential therapeutic approach to reducing the chemoresistance of pancreatic cancer $[16,28]$. However, the functional role of TAK1 in ovarian cancer chemoresistance is unknown. To define whether inhibition of TAK1 can sensitize ovarian cancer cells to cisplatin-induced cell apoptosis, XTT cell proliferation assay was performed in the TAK1 knockdown clones SK-sh1-KD3, SK-sh1-KD6, Acp-sh1KD1 and Acp-sh2-KD10. Notably, treatment of TAK1- depleted A2780cp clones (Acp-sh1-KD1 and Acp-sh2KD10) with cisplatin (MERK MILLIPORE, Billerica, MA; dissolved in DMSO), and TAK1-depleted SKOV3 clones (SK-sh1-KD3 and SK-sh1-KD6) with cisplatin led to a remarkable decrease in cell viability by $60 \%$ and $40 \%$ in Acp-sh1-KD1 and Acp-sh2-KD10, respectively $\left({ }^{*} P<0.01\right)$, and $40 \%$ in SK-sh1-KD3 $\left({ }^{*} P=0.028\right)$ as compared to their vector controls (Figure 4A). In addition, (5Z)-7-Oxozeaenol co-treatment with cisplatin decreased the cell viability of A2780cp and ES-2, which express high levels of TAK1 by $25 \%$ and $50 \%$, respectively $(* P<0.01)$ (Figure 4B). Moreover, focus formation assay confirmed that co-treatment of (5Z) -7-Oxozeaenol could cause less number and smaller size of colonies in both cell lines treated with cisplatin $\left({ }^{*} P<0.01\right)$ (Figure $4 C$ ). These findings confirm that the upregulation of TAK1 is involved in cisplatin-resistance of ovarian cancer cells.
A

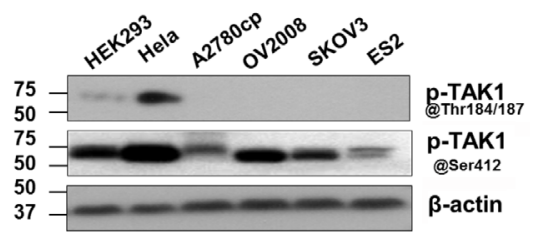

C

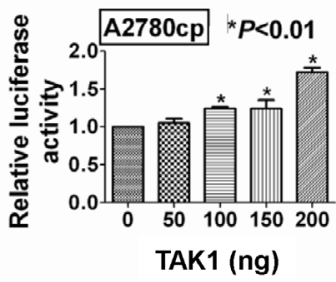

B

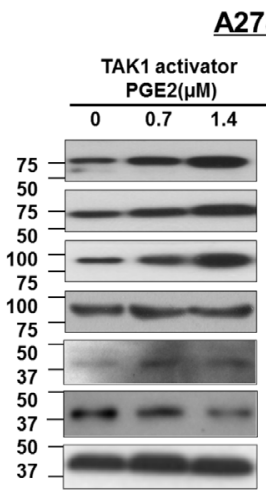

A2780cp

TAK1 inhibitor (5Z)-7-Oxozeaenol( $(\mu \mathrm{M})$

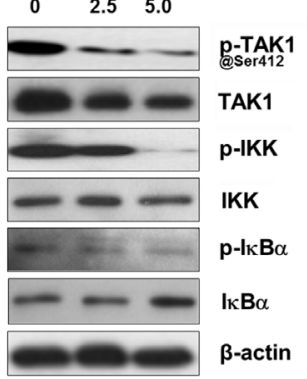

E A2780cp
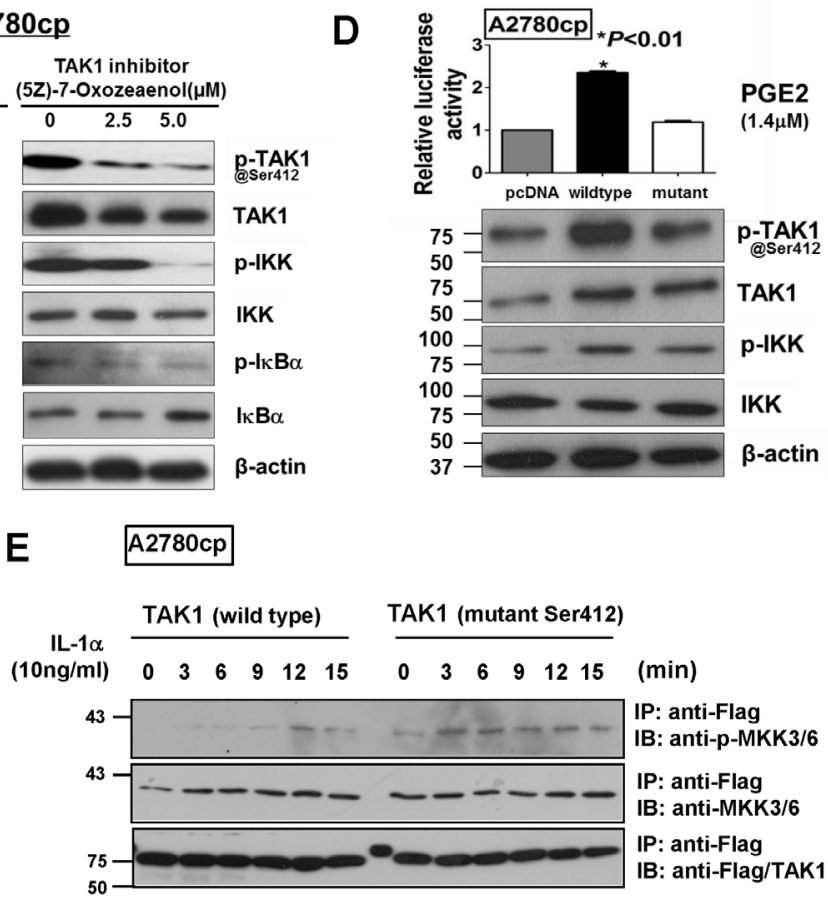

Figure 5: TAK1 exerts its functional effects through activation of NF - $\mathrm{B}$ pathways by phosphorylation at Ser412. (A) Western blot analysis demonstrated that p-TAK1 at Thr184/187 was just found in HEK293 and Hela cells only, while p-TAK1 at Ser412 was generally found in all cell types including ovarian cancer cell lines $(n=4)$. (B) Western blot analysis showed the phosphorylation of p-TAK1 (Ser412) was increased concomitantly with p-IKK (Ser180/181) and p-IKB (Ser32/36) as upon treatment of TAK1 activator, PGE2, in ovarian cancer cells, whereas treatment with TAK1 inhibitor, (5Z) -7-Oxozeaenol, completely diminished the phosphorylation of p-TAK1

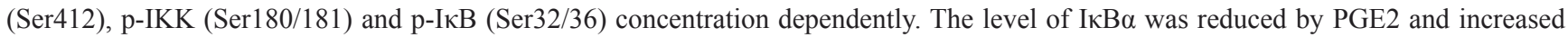
upon treatment of (5Z) -7-Oxozeaenol. Cells were treated by PGE2 or (5Z)-7-Oxozeaenol for 24 hours before harvested for Western blotting. The protein amount of loading was normalized by $\beta$-actin. (C) NF- $\kappa B$ luciferase reporter assay showed that TAK1 increased the luciferase activity of NF- $\mathrm{kB}$ in A2780cp concentration dependently ( $* * P<0.01)$. Co-treatment of TAK1 inhibitor, (5Z) -7-Oxozeaenol $\left(2.5 \mu \mathrm{M}\right.$ and $5.0 \mu \mathrm{M}$ for 24 hours), decreased the luciferase activity of NF- $\kappa \mathrm{B}$ in TAK1 transfected A2780cp cells ( $\left.{ }^{*} P<0.01\right)$. (D) Upon treatment of PGE2 $(1.4 \mu \mathrm{M})$, the point mutations at Ser412 of TAK1 had the similar expression level of TAK1 protein as wild type TAK1, however, the phosphorylation of p-TAK1 (Ser412) was totally abrogated. The protein amount of loading was normalized by $\beta$-actin. The above experiments were performed thrice independently. (E) TAK1 in vitro kinase assay. The pcDNA/Flag-TAK1 and pcDNA/FlagmutTAK1 were transfected into A2780cp cells respectively. After 48 hours, Human IL-1 $\alpha(10 \mathrm{ng} / \mathrm{ml})$ was used to treat the transfected cells with various time points. Both wild-type and mutant TAK1 were immunopreciptated (IP) from cell lysates, and TAK1 kinase activity was examined by evaluating the level of Phospho-MKK6 using immunoblotting (IB). The input of total MKK6 and Flag/TAK1 or Flag/mutant TAK1 were checked by immunoblotting using anti-MKK6 and anti-Flag respectively. 


\section{Ser412 phosphorylation is required for TAK1 to activate NF- $\mathrm{NB}$ signaling in ovarian cancer}

Since TAK1 was confirmed to be upregulated in ovarian cancer and its level well correlated with tumor progression, the molecular mechanism of TAK1 action deserves further investigation. The downstream effects of TAK1 are mediated via phosphorylation of multiple residues in its activation loop [18]. Our western blot analysis revealed that thephosphorylation of p-TAK1 (Thr184/187) was very low, whereas the phosphorylation of p-TAK1 (Ser412) was dominately expressed in all the ovarian cancer cell lines (Figure 5A). It has previously been reported that the NF- $\mathrm{KB}$ pathway is implicated in the regulation of cell migration and invasion [11, 12]. Since TAK1 takes many functional roles in ovarian cancer progression such as cell proliferation, migration/invasion and importantly, Kobayashi et al. has reported that the phosphorylation of the Ser412 residue in TAK1 could activate NF- $\kappa \mathrm{B}$ through induction of I $\mathrm{KB} \alpha$ degradation [21]. Therefore, we hypothesized that NF- $\mathrm{KB}$ signaling might also be activated by TAK1 via PKA-pathway mediated IкB $\alpha$ degradation in ovarian cancer cells. As expected, our western blot results showed that PGE2 could increase the phosphorylation of Ser412 in TAK1 that in turn, elevated the phosphorylation of p-IKK (Ser176/180) and $\mathrm{p}-\mathrm{I} \kappa \mathrm{B}(\mathrm{Ser} 32 / 36)$, and reduced the level of IкB $\alpha$ in A2780cp cells (Figure 5B). Conversely, treatment of A2780cp cells with the TAK1 inhibitor (5Z) -7-Oxozeaenol showed an opposite results to the use of PGE2 (Figure 5B). In addition, using NF- $\kappa \mathrm{B}$ luciferase reporter assay, NF- $\mathrm{KB}$ signaling activity was shown to increase from 1.2-fold to 2-fold when A2780cp cells were transiently transfected with the TAK1-expressing plasmid pCMV-HA-TAK from $0 \mathrm{ng}$ to $200 \mathrm{ng}(* P<0.01)$ (Figure 5C). In contrast, NF- $\kappa B$ signaling activity in A2780cp cells was reduced by $60 \%$ to $65 \%$ upon treatment with (5Z) -7-Oxozeaenol from $0 \mathrm{M}$ to $5.0 \mu \mathrm{M}\left({ }^{*} P<0.01\right)$ (Figure 5C).

To better understand whether phosphorylation at Ser412 is critically required for TAK 1 function, a mutant TAK1 plasmid (pCDH-TAK1-mut) was generated by PCR-based site-directed mutagenesis the Ser412 $\rightarrow$ Ala mutant TAK1 (Supplementary Figure S2) [21]. Western

B

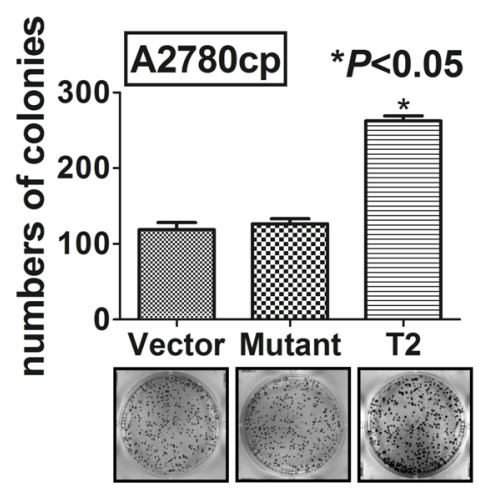

C

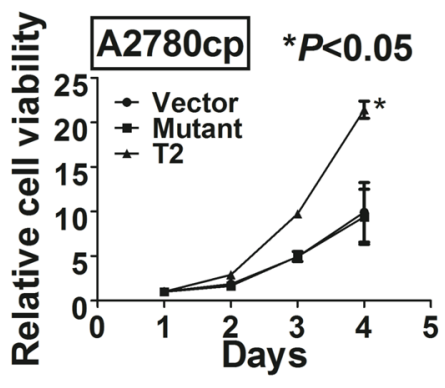

A2780cp (18h)
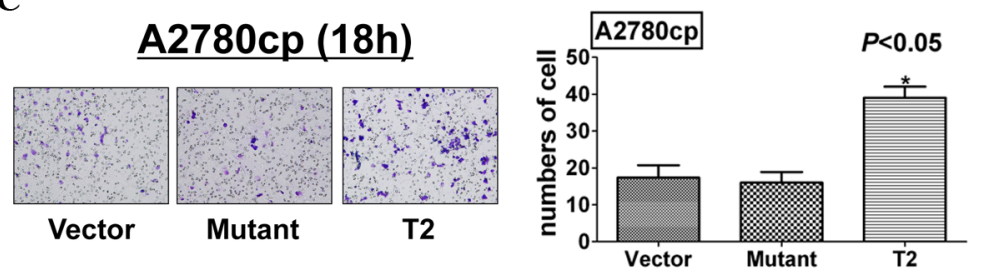

D
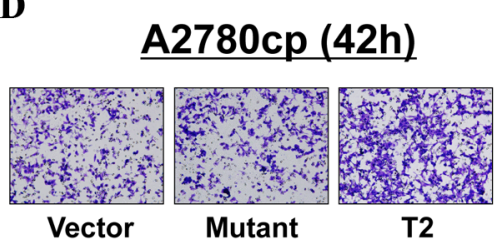

Figure 6: Mutation at Ser412 of TAK1 completely abrogates its function in ovarian cancer aggressiveness. (A) XTT cell proliferation assay, (B) focus formation, (C) Transwell migration, and (D) Transwell invasion assays demonstrated that the point mutation at Ser412 completely impaired the functional effects of TAK1 in promoting cell growth, cell migration and invasion as compared with wild-type TAK1 overexpressing A2780cp cells (T2) $\left({ }^{*} P<0.05\right)$. 
blot analysis confirmed that ectopic expression of the pCDH-TAK1-mut plasmid in A2780cp cells could not increase phosphorylation at Ser412 of TAK1 but had twofold less of the phosphorylation of p-IKK (Ser176/180) than that of using the wild-type TAK1 plasmid upon

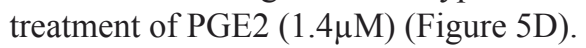

Accumulating evidence has suggested that TAK1 forms a complex with TAB1 and TAB2/3 at N- and C-termini respectively (Supplementary Figure S2) [29, 30], we questioned whether the phosphorylation at Ser412 residue in TAK1 could modulate the kinase activity of TAK1. Hence, we performed in vitro kinase assay for TAK1 activity according to Yang et al [28]. Upon induction of IL- $1 \alpha$ in A2780cp transfected with the wild-type TAK1 palsmid (pcDNA/Flag-TAK1) and the mutant TAK1 plasmid (pcDNA/Flag-mutTAK1), the immunoprecipitated wild-type TAK1 could remarkably phosphorylate its downstream target, MKK6, while the immunoprecipitated mutant TAK 1 had relatively lower capacity in phosphorylation of MKK6 (Figure 5E). This infers that the phosphorylation of TAK1 at Ser412 increase the kinase activity of TAK1 in ovarian cancer cells. Furthermore, pCDH-TAK1-Mut, when stably transfected into A2780cp, could not exert any increased higher capacities in cell proliferation, migration and invasion when compared with vector controls or as same as wildtype TAK1 overexpressing cells (Figure 6A-D). These data give the first report that phosphorylation of TAK1 at Ser412, instead of Thr184/187, is critically required for activation of the NF- $\mathrm{B}$ pathway and its oncogenic properties.

\section{DISCUSSION}

Recent studies have revealed that the personalized gene-targeted. cancer therapy shows promising results in improving the survival and quality of life of patients suffering advanced-stage and high-grade tumors [47]. Therefore, a better understanding of molecular mechanisms underlying ovarian cancer oncogenesis is urgently needed for developing this therapeutic approach. In this study, we have provided overwhelming evidence that TAK1 plays an oncogenic role in promoting cell proliferation, anchorage-independent growth ability, cell migration/invasion, and chemoresistance, as well as tumor growth/metastasis of ovarian cancer cells in both in vitro and in vivo models. Our findings indicate that TAK1 was significantly overexpressed in ovarian cancers, in particular high-grade and metastatic tumors. More importantly, we identify phosphorylation at Ser412 is critically required for TAK 1 in activating of $\mathrm{NF}-\kappa \mathrm{B}$ activity and its tumorigenic capacities in ovarian cancer cells.

Previous studies have reported that the increased $\mathrm{NF}-\kappa \mathrm{B}$ activity is involved in metastatic serous ovarian carcinoma and targeting $\mathrm{NF}-\kappa \mathrm{B}$ pathway is a promising therapeutic approach to high-grade metastatic ovarian cancer $[31,32]$. This indicates that NF- $\kappa B$ signaling acts as a master regulator in aggressive ovarian cancers. However, there is a lack of studies showing the upstream regulation of $\mathrm{NF}-\kappa \mathrm{B}$ signaling in carcinogenesis. In this study, we have revealed that TAK 1 is frequently found in high-grade tumors with metastatic tendency, suggesting that TAK1 shares similar functional properties as the NF$\kappa \mathrm{B}$ pathway in aggressive human cancers. On the other hand, previous studies have documented that TAK1 can upregulate the $\mathrm{NF}-\kappa \mathrm{B}$ signaling which in turn increases the chemoresistance of human cancers $[28,33,34]$. Hence, we postulate that TAK 1 promotes ovarian cancer cell growth, cell migration and invasion, as well as cisplatin-resistance via modulation of $\mathrm{NF}-\kappa \mathrm{B}$ signaling. Indeed, our results using Western blotting and NF- $\mathrm{B}$ promoter luciferase assays clearly show that TAK 1 is able to modulate NF- $\kappa B$ signaling activity, whereas either co-treatment with TAK1 inhibitor, (5Z) -7-Oxozeaenol, or shRNA-mediated TAK1 knockdown abrogates TAK1's effect on NF- $\kappa \mathrm{B}$ signaling activity in ovarian cancer cells.

TAK1 is a mitogen-activated protein kinase kinase kinase that can be activated by various upstream cytokines through formation of a protein-signaling complex which consists of a variety of proteins such as TAB1, TAB2/3 and TRAFs [35]. Thus, TAK1 can exert distinct functions in different cell types depending on the nature of the upstream inducing signals [35] This makes TAK1 a double-edged sword in different types of human cancers [36]. For instance, suppression of TAK1 is required to promote prostate cancer tumorgenesis [37], while activation of TAK1 leads to hepatocyte apoptosis in liver cancer through activation of JNK signaling and $\mathrm{NF}-\kappa \mathrm{B}$-independent function of NEMO, indicating that TAK1 is a tumor suppressor in some subtypes of human cancers [14, 37, 38]. On the other hand, TAK1 has been shown to enhance oncogenic capacities in liver cancer, lung cancer, pancreatic cancer and head and neck cancer

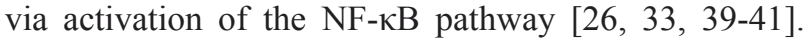
These evidences suggest that TAK1 plays dual roles in oncogenesis; tumor suppressor and oncogene, depending on the downstream signaling pathway that is activated and therefore dominating in a particular cancer cell type. In this study, we have demonstrated that NF- $\kappa \mathrm{B}$ signaling is indispensable for TAK1 function in ovarian cancer cells. It is well known that the phosphorylation of TAK1 can subsequently activate IKK leading to the activation of NF- $\kappa \mathrm{B}$ [14] and in cell metastasis of breast and renal cell cancers $[26,42]$. In fact, using the TAK1 inhibitor (5Z) -7-Oxozeaenol, as well as gain- and loss-of-function genetic modifications of TAK1, we have provided strong evidences to support that TAK1 is required for NF- $\mathrm{NB}$ activation in ovarian cancer. Hence, these findings point to TAK1 being an oncogenic gatekeeper for NF-кB signaling, which is a key signaling pathway in governing ovarian cancer tumorigenesis. 
It has been demonstrated that TAK1 kinase activity is regulated by multiple post-translational modifications of TAK1 and TABs [43]. Many phosphorylation sites had been identified previously, such as Ser192, Thr178, Thr184/187 and Ser412 [18-21]. Ser192 autophosphorylaion was first identified to be involved in IL-1 induced activation of NF- $\kappa B$ through the interaction of TAB1 at N-terminus of TAK1 kinase domain [18]. Dual phosphorlation of Thr178 and Thr184 was required for IL-1- and IL-6-mediated activation of both the NF$\kappa \mathrm{B}$ and JNK pathways [19]. In this study, we have surprisingly found that Thr184/187 of TAK1 is not the core phosphorylated sites in ovarian cancer cells. Instead, we identify the phosphorylation site at the Ser412 residue as being critically required for TAK1 in mediating NF$\kappa \mathrm{B}$ activity in ovarian cancer cells. Although the Ser412 residue is not in the kinase domain of TAK1, it has been shown to be interacted with TAB2 at the C-terminus of TAK1 and could be phosphorylated by PKA pathway [21]. cAMP/PKA signaling is an oncogenic signaling and is involved in ovarian cancer progression [44] [45]. Importantly, cAMP/PKA signaling has been reported to promote $\mathrm{NF}-\kappa \mathrm{B}$ activity[46, 47]. Indeed, in consistent with the previous finding of Kabayashi et al. [21], our data showed that the increased phosphorylation of Ser412 by treatment of PGE2 led to the increased degradation of $\mathrm{I} \kappa \mathrm{B} \alpha$ (elevated $\mathrm{p}-\mathrm{I} \kappa \mathrm{B}(\mathrm{Ser} 32 / 36))$ and increased phoshphorylation of p-IKK (Ser176/180) (NF-кB activity). This has been further confirmed using NF-kB luciferase reporter assay. In contrast, mutation by Ser412 $\rightarrow$ Ala aboragated the capacity of TAK1 in promoting $\mathrm{NF}-\kappa \mathrm{B}$ activity as well as its associated oncogenic properties in ovarian cancer cells. On the other hand, it has been shown that the phosphorylation of TAK1 Ser412 by PKA may influence the signaling complex formation (TAK1-TAB1TAB2/TAB3) induced by the various ligands including IL- $1 \alpha$ in Ser192 of TAK1 $[18,21]$. To examine whether the phosphorylation of Ser412 affects the complex confirmation as well as the kinase activity of TAK1, an in vitro TAK1 kinase was performed to examine the capacity of the wild-type or the Ser412 mutant TAK1 in modulating TAK1 activity under IL- $1 \alpha$ induction. As expected, we found that Ser412 phosphorylation has not only activated $\mathrm{NF}-\kappa \mathrm{B}$ through degradation of $\mathrm{I} \kappa \mathrm{B} \alpha$ but also alters IL$1 \alpha$-induced TAK1 activity. However, we still don't know how the phosphorylation of Ser412 affects the signaling complex formation (TAK1-TAB1-TAB2/TAB3) and the kinase activity. Hence, further study in examining the underlying mechanism is warranted. However, to the best of our knowledge, this is the first report showing that the phosphorylation of Ser412 residue in TAK1 is essential for the activation of NF- $\kappa \mathrm{B}$ signaling in ovarian cancer cells.

In conclusion, our findings suggest that the upregulation of TAK1 and the phosphorylation at Ser412 residue in TAK1 is required for enhancing NF$\kappa \mathrm{B}$-dependent oncogenic capacities in aggressive high- grade ovarian cancer. Therefore, targeting TAK1 may be a potential therapeutic approach in the personalized molecular therapy in ovarian cancer.

\section{MATERIALS AND METHODS}

\section{Ovarian cancer samples and cell lines}

A total of 88 tumor samples surgically resected from primary ovarian cancer patients and 48 normal ovary samples from benign diseases were randomly selected for this study. The histologic subtypes and disease stages of the tumors were classified according to International Federation of Gynecology and Obstetrics criteria. The use of clinical specimens was approved by the local institutional ethics committee (IRB no. UW11-298). Four ovarian cancer cell lines (high-grade serous subtype); A2780cp, (kindly provided from Professor Benjamin Tsang, The University of Ottawa), SKOV3 and OVCA429 (ATCC, Rockville, MD, USA) [48], one ovarian cancer cell line (clear cell subtype), ES-2, (ATCC), as well as HEK293 cells were cultured in Dulbecco's modified Eagle medium (DMEM) (Gibco-BRL, Gaithersburg, MD) with 10\% Fetal Bovine Serum (FBS) (Gibco-BRL, Gaithersburg, MD), penicillin and streptomycin (100 units $/ \mathrm{ml}$ ) at $37^{\circ} \mathrm{C}$ in incubator with $5 \% \mathrm{CO}_{2}$. The cell line authentication was done by in-house STR DNA profiling analysis. Two pairs of primary cultured cells from the same patients from both omentum (OMC) and ovaries (OVC) were obtained in Queen Mary Hospital under human ethics (HKU/HA HKW IRB number: UW 11298). The primary culture cells were incubated with a mix medium of MCD (Invitrogen) and 199 (Invitrogen) with a proportion of 1:1. All the experiments were performed after 10 days incubation.

\section{Plasmids and Cell Transfection}

Stable TAK1-overexpressing clones were generated by either transfection of pCMV-HA-TAK (kindly provided by Kuni Matsymoto Lab, Acp-T2 and Acp-T3) or pCDHTag-1-TAK1 lentiviral plasmid (429-C12 and 429-C13) constructed by PCR amplification of the TAK1 ORF from pCMV-HA-TAK followed by subcloning into the pCDHCMV-MCS-EF1-copGFP (SBI). TAK1 shRNAi constructs (HuSHTM Company, Japan) were used to knockdown endogenous TAK1 in TAK1-overexpressing ovarian cancer cell lines. Stable mutant Ser412 TAK1 clones were generated by transfection of A2780cp cells with the pCDH-TAK1-mut plasmid, which was constructed by PCR-based site-directed mutagenesis of Ser 412 to Ala 412 using designed primers according to Kobayashi et al. [21]. GFP-luminescence labeled stable clones were generated by lenti-virus transfection of Lenti-reporter 
plasmid, CMV-GFP-T2A-Luciferase (BLIV101PA-1) (System Biosciences, Mountain view, CA).

\section{Quantitative Reverse Transcription-Polymerase Chain Reaction (qPCR)}

qPCR was performed using the oligonucleotides/ TagMan probes provided for TagMan Gene Expression Assays (Applied Biosystems, Foster City, CA) (TAK1, Assay ID: HS01105682 m1; 18S, Catalog Number: 4310893E) in an ABI 7500 system according to the manufacture's instruction. Expression levels of target genes were quantified against endogenous 185 level using the comparative $\mathrm{C}_{\mathrm{T}}$ method. The 7500 System SDS Software was used for data analysis.

\section{Western blot and Immunohistochemical (IHC) analyses}

For Western blot analysis, cells were lysed with cell lysis buffer (Cell Signaling Technology, Danvers, MA) containing protease inhibitor cocktail (Roche, Indianapolis, IN) and phenylmethylsulfonyl fluoride (Sigma, St. Louis, MO). Samples were resolved by SDSPAGE and electroblotted onto Immobilon-P Transfer Membrane (Merk Millipore, Billerica, MA). Blots were blocked with $5 \%$ skim milk, followed by incubation with antibodies against TAK1, Phospho-TAK1 (Ser412), Phospho-TAK1 (Thr184/187), Phospho-IKK $\alpha / \beta$ (Ser180/181), IKK, Phospho- ІкB $\alpha$ (Ser32/36), ІкB $\alpha$, Phospho-MKK3 (Ser189)/MKK6 (Ser207) (Cell Signaling Technology, Danvers, MA); MKK6 (R\&D Systems, Minneapolis, MN); GFP (Santa Cruz Biotechnology, Santa Cruz, CA) or $\beta$-actin (Sigma, St. Louis, MO). Blots were then incubated with goat anti-rabbit or antimouse secondary antibodies that are conjugated with horseradish peroxidase (Amersham Pharmacia Biotech, Piscataway, NJ) and signals visualized by enhanced chemiluminescence .

Immunohistochemical staining for TAK1 was performed on an ovarian cancer tissue array (OVC1021; Pantomics, Inc.). The section was immunostained with primary polyclonal anti-TAK1 antibody (Cell signaling Technology, Danvers, MA, USA) in 1:50 dilution. For negative controls, the primary antibody was replaced with TBS. The immunoreactivity score for each case was calculated as previously protocol [49].

\section{Luciferase reporter assay}

Luciferase Reporter Assay was performed using the DLR Assay kit (Promega, Madison, WI) according to the manufacturer's instructions. Cells were cultured on 6-well plates and were transfected with plasmids two days before the luciferase assay.

\section{In vitro Kinase assay}

To examine the TAK1 activity, an in vitro kinase assay was modified from Yang et al. [28]. The wild type TAK1 and mutant TAK1 from pCMV-HA-TAK and pCDH-TAK1-mut plasmids were subcloned into pcDNA3.1(+)/3xFlag vector to generate pcDNA/FlagTAK1 and pcDNA/Flag-mutTAK1. Both plasmids were transfected into A2780cp cells, respectively an cell lysates were harvested after 48 hours and treated with Human IL-1 $\alpha$ (Peprotech, Rocky Hill, NJ). Cell lysates were prepared by Cell Lysis Buffer (Cell Signaling) and immunoprecipitated with anti-Flag (Sigma) followed by overnight incubation with protein $\mathrm{A} / \mathrm{G}$-conjugated beads (Santa Cruz) at $4^{\circ} \mathrm{C}$. After 3 times of washing using Cell Lysis Buffer, an in vitro kinase reaction was performed at $30^{\circ} \mathrm{C}$ for $90 \mathrm{~min}$ in $20 \mu \mathrm{l}$ Kinase Buffer (Cell Signaling) containing ATP $(200 \mu \mathrm{M})$ and $1 \mu \mathrm{g}$ of unactive MKK6/ SKK3 protein (Millipore, Billerica, MA). The activities of wild type TAK 1 and mutant TAK 1 were determined by the levels of phospho-MKK6 using Western blotting.

\section{In vitro functional assays}

Cell proliferation kit (XTT) (Roche, Indianapolis, IN, USA) was used to measure cell viability according to the manufacturer's manual [50]. Focus formation assay was performed as a more long-term proliferation assay and soft agar assay was performed to detect cell anchorage-independent growth ability. Wound healing assay and a Cell Migration Assay kit (MILLIPORE, Cat. No. ECM508) were used to detect cell migration ability. Quantification of cell invasion was performed using QCM ${ }^{\mathrm{TM}}$ 24-Well Colorimetric Cell Invasion Assay Kit (Chemicon International, Temecula, CA, USA). All experiments were done in triplicates and results were presented as the mean $\pm \mathrm{SD}$.

\section{Tumor xenograft mouse model}

A2780cp-sh1-KD1 and its scrambled control cells at a concentration of $1 \times 10^{6}$ cells $/ 100 \mu 1$ were injected subcutaneously into 5-week female BALB/c nude mice. For Acp-sh1-KD1 and vector control injection, tumor formation in nude mice $(\mathrm{n}=5)$ was monitored for every 3 days since Day 3. For the A2780cp cell injection group, the TAK1 inhibitor, (5Z) -7-Oxozeaenol $(16 \mathrm{mg} / \mathrm{kg})$ (Sigma) (in carrier solution which consists of $10 \%$ DMSO in PBS), was injected into the mice in peritoneal every three days., was administered by intraperitoneal (i.p.) injections for every two days with total of 7 injections into five mice from Day 3. As a control group, the carrier 
solution only was administrated i.p. for the same time of treatment. Tumor sizes were measured using slide calipers and were calculated by the following formula: volume $=(\text { width })^{2} \mathrm{x}$ length $\mathrm{x} \pi / 6$. Tumor growth curves were plotted from the mean volume \pm SEM of tumors from 5 mice. Side effects such as body weight changes were monitored closely. For in vivo mouse model of metastasis, the pure GFP-luminescence tagged SKOV3 cells $\left(1 \times 10^{6}\right.$ cells $/ 100 \mu \mathrm{l}$ ) were mixed with $100 \mu \mathrm{l}$ Matrigel Matrix (BD Biosciences, San Jose, CA) and intraperitoneally injected into 6 nude mice. TAK1 inhibitor, (5Z) -7-Oxozeaenol $(16 \mathrm{mg} / \mathrm{kg})$ (in carrier solution), was administered i.p. for every three days with total 5 injections into 3 mice. For the control group, the carrier solution was injected i.p. only. The ovarian cancer cells (with GFP-labelled) metastasized to livers of mice were analyzed by biofluorscence imaging. Both bioluminescence and biofluorescence imagings were performed using the Xenogen IVIS 100 system and analyzed using Living Imaging ${ }^{(\mathrm{R})}$, version 2.50.1. All the animal experiments were approved by the University of Hong Kong Committee on the Use of Live Animals in Teaching and Research (CULATR No. 2053-09).

\section{Statistical analysis}

Receiver operating characteristic (ROC) curve was used to determine cut-off points for qPCR and IHC results. The clinicopathological analysis between the expression of TAK1 and clinical parameters was analyzed by Crosstabs and Pearson Chi-Square test. The Student's $t$-test was used to analyze cell viability, migration/invasion and in vivo tumor growth results. Statistical analyses were performed using the SPSS 13.0 software (SPSS). $P$-values of less than 0.05 were considered significant in all tests. All data were expressed as mean $\pm \mathrm{SD}$.

\section{ACKNOWLEDGEMENTS}

We thank for Dr. Kuni Matsymoto Lab (Kanazawa University, Japan) for providing pCMV-HA-TAK plasmid, and Prof. Benjamin Tsang (Department of Obstetrics and Gynaecology, The University of Ottawa) for providing ovarian cancer cell line, A2780cp.

\section{Financial support}

This study was supported by the Hong Kong Research Grants Council General Research Fund (HKU 761213M), and Wong Check She Charitable Foundation.

\section{Disclosure of Potential Conflicts of Interest}

All authors have no potential conflicts of interest were disclosed.

\section{Statement of author contributions}

PC and DC designed research; PC, LS, VL, HT, IL and DC performed the experiments; DC, TL and KY contributed new reagents-analytic tools; PC, DC, JY, KC and $\mathrm{HN}$ analyzed and interpreted data; $\mathrm{PC}$ and $\mathrm{DC}$ wrote the manuscript; DC and HN study supervision All authors were involved in editing the manuscript and had final approval of the submitted and published versions.

\section{REFERENCES}

1. Jemal A, Bray F, Center MM, Ferlay J, Ward E and Forman D. Global Cancer Statistics. Ca-a Cancer Journal for Clinicians. 2011; 61(2):69-90.

2. Modugno F and Edwards RP. Ovarian cancer: prevention, detection, and treatment of the disease and its recurrence. Molecular mechanisms and personalized medicine meeting report. Int J Gynecol Cancer. 2012; 22(8):S45-57.

3. Stakleff KS, Sloan T, Blanco D, Marcanthony S, Booth TD and Bishayee A. Resveratrol Exerts Differential Effects in Vitro and in Vivo against Ovarian Cancer Cells. Asian Pacific Journal of Cancer Prevention. 2012; 13:1333-1340

4. Rahman M, Hoh B, Kohler N, Dunbar EM and Murad GJ. The future of glioma treatment: stem cells, nanotechnology and personalized medicine. Future Oncol. 2012; 8(9):11491156.

5. Bellmunt J and Petrylak DP. New therapeutic challenges in advanced bladder cancer. Semin Oncol. 2012; 39(5):598607.

6. Van Allen EM and Pomerantz M. Moving toward personalized medicine in castration-resistant prostate cancer. Urol Clin North Am. 2012; 39(4):483-490.

7. Hekeler E, Zoller WG, Wiedorn KH and Bosse A. [The change of pathology in the era of personalized medicine using the example of discordant KRAS mutational status in metastasized colorectal carcinoma]. Dtsch Med Wochenschr. 2012; 137(45):2327-2331.

8. Yang G, Xiao X, Rosen DG, Cheng X, Wu X, Chang B, Liu G, Xue F, Mercado-Uribe I, Chiao P, Du X and Liu J. The biphasic role of NF-kappaB in progression and chemoresistance of ovarian cancer. Clin Cancer Res. 2011; 17(8):2181-2194.

9. White KL, Rider DN, Kalli KR, Knutson KL, Jarvik GP and Goode EL. Genomics of the NF-kappa B signaling pathway: hypothesized role in ovarian cancer. Cancer Causes \& Control. 2011; 22(5):785-801.

10. Hernandez L, Hsu SC, Davidson B, Birrer MJ, Kohn EC and Annunziata CM. Activation of NF-kappaB signaling by inhibitor of NF-kappaB kinase beta increases aggressiveness of ovarian cancer. Cancer Res. 2010; 70(10):4005-4014.

11. Mabuchi S, Ohmichi M, Nishio Y, Hayasaka T, Kimura A, Ohta T, Saito M, Kawagoe J, Takahashi K, YadaHashimoto N, Sakata M, Motoyama T, Kurachi H, Tasaka 
$\mathrm{K}$ and Murata Y. Inhibition of NFkappaB increases the efficacy of cisplatin in in vitro and in vivo ovarian cancer models. J Biol Chem. 2004; 279(22):23477-23485.

12. Chen SS, Michael A and Butler-Manuel SA. Advances in the treatment of ovarian cancer: a potential role of antiinflammatory phytochemicals. Discov Med. 2012; 13(68):7-17.

13. Taniguchi F, Harada $\mathrm{T}$, Miyakoda H, Iwabe $\mathrm{T}$, Deura I, Tagashira Y, Miyamoto A, Watanabe A, Suou K, Uegaki $\mathrm{T}$ and Terakawa N. TAK1 activation for cytokine synthesis and proliferation of endometriotic cells. Mol Cell Endocrinol. 2009; 307(1-2):196-204.

14. Inokuchi S, Aoyama T, Miura K, Osterreicher CH, Kodama Y, Miyai K, Akira S, Brenner DA and Seki E. Disruption of TAK1 in hepatocytes causes hepatic injury, inflammation, fibrosis, and carcinogenesis. Proc Natl Acad Sci U S A. 2010; 107(2):844-849.

15. Omori E, Matsumoto K, Zhu S, Smart RC and NinomiyaTsuji J. Ablation of TAK1 upregulates reactive oxygen species and selectively kills tumor cells. Cancer Res. 2010; 70(21):8417-8425.

16. Melisi D, Xia Q, Paradiso G, Ling J, Moccia T, Carbone C, Budillon A, Abbruzzese JL and Chiao PJ. Modulation of pancreatic cancer chemoresistance by inhibition of TAK1. J Natl Cancer Inst. 2011; 103(15):1190-1204.

17. Singh A, Sweeney MF, Yu M, Burger A, Greninger P, Benes C, Haber DA and Settleman J. TAK1 inhibition promotes apoptosis in KRAS-dependent colon cancers. Cell. 2012; 148(4):639-650.

18. Kishimoto K, Matsumoto K and Ninomiya-Tsuji J. TAK1 mitogen-activated protein kinase kinase kinase is activated by autophosphorylation within its activation loop. J Biol Chem. 2000; 275(10):7359-7364.

19. Yu Y, Ge N, Xie M, Sun W, Burlingame S, Pass AK, Nuchtern JG, Zhang D, Fu S, Schneider MD, Fan J and Yang J. Phosphorylation of Thr-178 and Thr-184 in the TAK1 T-loop is required for interleukin (IL)-1-mediated optimal NFkappaB and AP-1 activation as well as IL-6 gene expression. J Biol Chem. 2008; 283(36):24497-24505.

20. Singhirunnusorn P, Suzuki S, Kawasaki N, Saiki I and Sakurai H. Critical roles of threonine 187 phosphorylation in cellular stress-induced rapid and transient activation of transforming growth factor-beta-activated kinase 1 (TAK1) in a signaling complex containing TAK1-binding protein TAB1 and TAB2. J Biol Chem. 2005; 280(8):7359-7368.

21. Kobayashi Y, Mizoguchi T, Take I, Kurihara S, Udagawa $\mathrm{N}$ and Takahashi N. Prostaglandin E2 enhances osteoclastic differentiation of precursor cells through protein kinase A-dependent phosphorylation of TAK1. J Biol Chem. 2005; 280(12):11395-11403.

22. Bhattacharyya S, Borthakur A, Anbazhagan AN, Katyal S, Dudeja PK and Tobacman JK. Specific effects of BCL10 Serine mutations on phosphorylations in canonical and noncanonical pathways of NF-kappa B activation following carrageenan. American Journal of Physiology-Gastrointestinal and Liver Physiology. 2011; 301(3):G475-G486.

23. Aurelian L, Laing JM and Lee KS. H11/HspB8 and Its Herpes Simplex Virus Type 2 Homologue ICP10PK Share Functions That Regulate Cell Life/Death Decisions and Human Disease. Autoimmune Dis. 2012; 2012:395329.

24. Lengyel E. Ovarian cancer development and metastasis. Am J Pathol. 2010; 177(3):1053-1064.

25. Sood AK, Coffin JE, Schneider GB, Fletcher MS, DeYoung BR, Gruman LM, Gershenson DM, Schaller MD and Hendrix MJ. Biological significance of focal adhesion kinase in ovarian cancer: role in migration and invasion. Am J Pathol. 2004; 165(4):1087-1095.

26. Safina A, Sotomayor P, Limoge M, Morrison C and Bakin AV. TAK1-TAB2 signaling contributes to bone destruction by breast carcinoma cells. Mol Cancer Res. 2011; 9(8):1042-1053.

27. Safina A, Ren MQ, Vandette E and Bakin AV. TAK1 is required for TGF-beta 1-mediated regulation of matrix metalloproteinase-9 and metastasis. Oncogene. 2008; 27(9):1198-1207.

28. Yang Y, Xia F, Hermance N, Mabb A, Simonson S, Morrissey S, Gandhi P, Munson M, Miyamoto S and Kelliher MA. A cytosolic ATM/NEMO/RIP1 complex recruits TAK1 to mediate the NF-kappaB and p38 mitogenactivated protein kinase (MAPK)/MAPK-activated protein 2 responses to DNA damage. Mol Cell Biol. 2011; 31(14):2774-2786.

29. Kanayama A, Seth RB, Sun L, Ea CK, Hong M, Shaito A, Chiu YH, Deng L and Chen ZJ. TAB2 and TAB3 activate the NF-kappaB pathway through binding to polyubiquitin chains. Mol Cell. 2004; 15(4):535-548.

30. Liu Q, Busby JC and Molkentin JD. Interaction between TAK1-TAB1-TAB2 and RCAN1-calcineurin defines a signalling nodal control point. Nat Cell Biol. 2009; 11(2):154-161.

31. Kleinberg L, Dong HP, Holth A, Risberg B, Trope CG, Nesland JM, Florenes VA and Davidson B. Cleaved caspase-3 and nuclear factor-kappaB p65 are prognostic factors in metastatic serous ovarian carcinoma. Hum Pathol. 2009; 40(6):795-806.

32. Annunziata CM, Stavnes HT, Kleinberg L, Berner A, Hernandez LF, Birrer MJ, Steinberg SM, Davidson B and Kohn EC. Nuclear factor kappaB transcription factors are coexpressed and convey a poor outcome in ovarian cancer. Cancer. 2010; 116(13):3276-3284.

33. Carbone $\mathrm{C}$ and Melisi D. NF-kappaB as a target for pancreatic cancer therapy. Expert Opin Ther Targets. 2012; 16 Suppl 2:S1-10.

34. Antoon JW, White MD, Slaughter EM, Driver JL, Khalili HS, Elliott S, Smith CD, Burow ME and Beckman BS. Targeting NFkB mediated breast cancer chemoresistance through selective inhibition of sphingosine kinase-2. Cancer 
Biol Ther. 2011; 11(7):678-689.

35. Delaney JR and Mlodzik M. TGF-beta activated kinase-1: new insights into the diverse roles of TAK1 in development and immunity. Cell Cycle. 2006; 5(24):2852-2855.

36. Thakur N, Sorrentino A, Heldin $\mathrm{CH}$ and Landstrom M. TGF-beta uses the E3-ligase TRAF6 to turn on the kinase TAK1 to kill prostate cancer cells. Future Oncol. 2009; 5(1):1-3.

37. Wu M, Shi L, Cimic A, Romero L, Sui G, Lees CJ, Cline JM, Seals DF, Sirintrapun JS, McCoy TP, Liu W, Kim JW, Hawkins GA, Peehl DM, Xu J and Cramer SD. Suppression of Tak1 promotes prostate tumorigenesis. Cancer Res. 2012; 72(11):2833-2843.

38. Malato $\mathrm{Y}$ and Willenbring $\mathrm{H}$. The MAP3K TAK1: a chock block to liver cancer formation. Hepatology. 2010; 52(4):1506-1509.

39. Ahmed N, Zeng M, Sinha I, Polin L, Wei WZ, Rathinam C, Flavell R, Massoumi R and Venuprasad K. The E3 ligase Itch and deubiquitinase Cyld act together to regulate Tak1 and inflammation. Nat Immunol. 2011; 12(12):1176-1183.

40. Freudlsperger C, Bian Y, Contag Wise S, Burnett J, Coupar J, Yang X, Chen Z and Van Waes C. TGF-beta and NFkappaB signal pathway cross-talk is mediated through TAK1 and SMAD7 in a subset of head and neck cancers. Oncogene. 2012.

41. Vucur M, Roderburg C, Bettermann K, Tacke F, Heikenwalder M, Trautwein C and Luedde T. Mouse models of hepatocarcinogenesis: what can we learn for the prevention of human hepatocellular carcinoma? Oncotarget. 2010; 1(5):373-378.

42. Ozbek E, Aliskan T, Otunctemur A, Calik G, Cakir S, Dursun $\mathrm{M}$ and Somay A. Comparison of tumor grade and stage with nuclear factor kappa $\mathrm{b}$ and p38 mitogene activated protein kinase expressions in renal cell cancer. Archivio italiano di urologia, andrologia : organo ufficiale [di] Societa italiana di ecografia urologica e nefrologica / Associazione ricerche in urologia. 2012; 84(2):53-60.

43. Sakurai H. Targeting of TAK1 in inflammatory disorders and cancer. Trends Pharmacol Sci. 2012; 33(10):522-530.

44. McDaid HM, Cairns MT, Atkinson RJ, McAleer S, Harkin DP, Gilmore P and Johnston PG. Increased expression of the RIalpha subunit of the cAMP-dependent protein kinase A is associated with advanced stage ovarian cancer. Br J Cancer. 1999; 79(5-6):933-939.

45. Al-Alem LF, McCord LA, Southard RC, Kilgore MW and Curry TE, Jr. Activation of the PKC pathway stimulates ovarian cancer cell proliferation, migration, and expression of MMP7 and MMP10. Biology of reproduction. 2013; 89(3):73.

46. Gerlo S, Kooijman R, Beck IM, Kolmus K, Spooren A and Haegeman G. Cyclic AMP: a selective modulator of NF-kappaB action. Cellular and molecular life sciences : CMLS. 2011; 68(23):3823-3841.

47. Gao N, Hibi Y, Cueno M, Asamitsu K and Okamoto T.
A-kinase-interacting protein 1 (AKIP1) acts as a molecular determinant of PKA in NF-kappaB signaling. J Biol Chem. 2010; 285(36):28097-28104.

48. Fung FK, Chan DW, Liu VW, Leung TH, Cheung AN and Ngan HY. Increased expression of PITX2 transcription factor contributes to ovarian cancer progression. PLoS One. 2012; 7(5):e37076.

49. Chan DW, Hui WW, Cai PC, Liu MX, Yung MM, Mak CS, Leung TH, Chan KK and Ngan HY. Targeting GRB7/ ERK/FOXM1 signaling pathway impairs aggressiveness of ovarian cancer cells. PLoS One. 2012; 7(12):e52578.

50. Lok GT, Chan DW, Liu VW, Hui WW, Leung TH, Yao KM and Ngan HY. Aberrant activation of ERK/FOXM1 signaling cascade triggers the cell migration/invasion in ovarian cancer cells. PLoS One. 2011; 6(8):e23790. 